\title{
Overview of Network Slicing: Business and Standards Perspective for Beyond 5G Networks
}

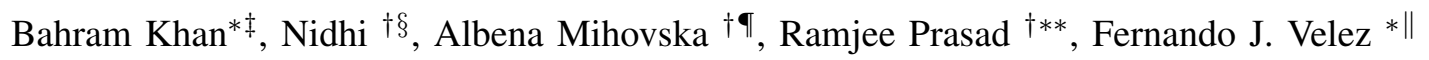 \\ *Instituto de Telecomunicações and Universidade da Beira Interior, \\ Faculdade de Engenharia, Departamento de Engenharia Electromecânica Covilhã, Portugal \\ ${ }^{\dagger} \mathrm{AU}$ - Department of Business Development and Technology, Aarhus University, Herning, Denmark

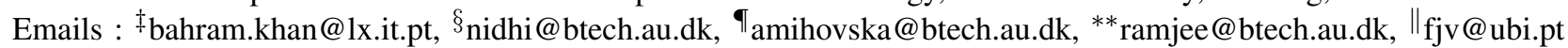

\begin{abstract}
The deployment of fifth-generation wireless communications (5G) networks brought a significant difference in the data rate and throughput to the wireless systems. It ensures ultralow latency and high reliability. In particular, Network Slicing (NS), one of the enablers for the 5G phase-II and beyond, has opened enormous opportunities for the Communications Service Provider (CSPs). NS allows CSPs to create independent virtual networks in the same physical network to guarantee high service levels. This paper provides an overview of the advances in NS from the perspective of the business opportunities and associated standardization activities. Standardization is critical in research as it intends to maintain interoperability among multi-vendor scenarios in telcos. We emphasize highlighting the technical facets of slicing within the business implementation and industry standardization process. Additionally, we address the application of Artificial Intelligence (AI) and Machine Learning (ML) to NSenabled future networks deployments. A set of use cases and the underlying specific requirements challenges are discussed as well. Finally, future research directions are addressed in detail.
\end{abstract}

Index Terms-Network Slicing, CSPs, AI, ML, Standards, SDOs

\section{INTRODUCTION}

The evolution of the mobile and wireless networks sector enabled by emerging 5G New Radio (NR) technologies and massively connected devices supports a significant increase in data traffic demand. As a result, new business models and services are available, e.g., supported by ultra-low latency and ultra-high throughput networks. The need for diverse applications and seamless connectivity involves critical requirements and implies supporting on-demand services. With the roll-out of fifth-generation $(5 \mathrm{G})$ wireless networks, Network Slicing (NS) evolved as a fundamental feature to facilitate segmented layers of networks in addition to the base network architecture [1].

In reality, NS provides a paradigm shift from the conventional approach towards traffic and network management [2]. It allows virtual logical network layers capable of enabling all the functionalities of a shared physical network. Furthermore, it sub-divides the network into several isolated virtual networks leading to a dedicated channel to provide resources to serve the user demands. Thus, slicing empowers conventional networks to support a wide range of use cases and business models.
Additionally, while enabling enhanced service quality, NS supports tailor-made user-specific solutions. For instance, the latency requirement for emergency services is more stringent than for agriculture-based applications (to maintain crop health). Thus, it will make current networks dynamic, flexible and scalable whilst accommodating growing demand, from various applications, with diverse requirements.

Unlike conventional networks, 5G networks are enabled with networking slicing. 5G evolution opens up many services and use cases. New physical networks are not required anymore to facilitate dedicated service. As proposed by the Third Generation Partnership Project (3GPP), the introduction of NS is established in the framework of release 15 [3] and is regularly updated for the required technical details and enhancements. 3GPP has specified that, for Communications Service Provider (CSPs), NS is significant for creating new services and generating new business models. NS allows CSPs to create multiple virtual slices to encompass colossal traffic increase and specific user requirements. As a consequence of this evolution, Working Groups from various Standard Development Organizations (SDOs) entered into force to support a multi-vendor landscape that develops NS specifications and guidelines.

CSPs benefit by implementing network orchestration, the automated communication among various entities on and across the network to meet network and user requirements. It sets guidelines to establish connections through the network while offering services with the associated Service Level Agreements (SLAs) [4].

In the scope of this paper, we provide an overview of new services and use case scenarios by the CSPs together with the presentation of associated standardization activities. In addition, we shed light on the implementation of Artificial Intelligence (AI) and Machine Learning (ML) techniques for NS and management as a whole.

The remaining of the paper is organized as follows. Section II discusses the state-of-the-art and Section III describes the standardization activities addressing the NS. In Section IV, the challenges and requirements are addressed. Finally, Section $\mathrm{V}$ presents the main conclusions of this work and addresses 
topics for further research.

\section{STATE-OF-THE-ART}

A network slice (often referred to as "5G Slice") incorporates the Network Functions (NF) and settings that encompass the supported use case or applications being served. It facilitates resources on-demand by incorporating existing virtualization and computing techniques. Slices make the resources modular while introducing flexibility into the network. In [5], authors have defined different layers of $5 \mathrm{G}$ slices, as shown in Figure 1.

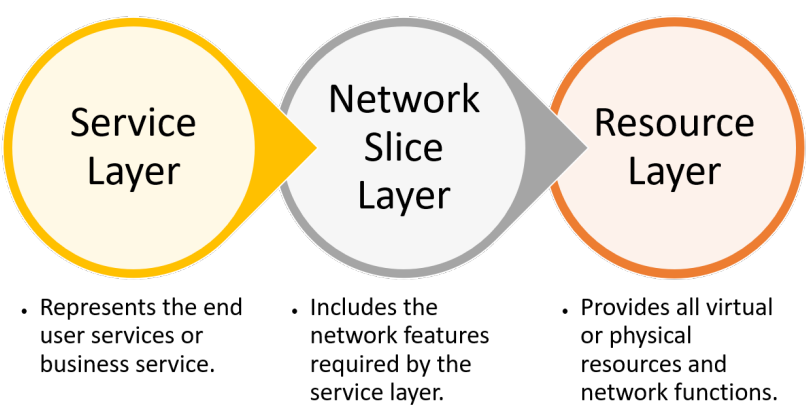

Fig. 1. Different Layers of Network Slicing

The two key enablers of Network Slicing are described as follow:

a) Virtualization Technologies: Virtualization facilitates resource sharing on the 5G Slice and removes dependencies on dedicated hardware. As the resources are independent of the physical hardware, slices are easy to deploy and manage as a modular block on a 5G network [5]. Interfacing the independent modules of resources is critical, and needs assistance for resource allocation purposes.

b) Management and Orchestration: Automated orchestration and management techniques help regulate and manage many network slices in a complex environment. Besides, according to [6], it facilitates network slice management functions.

\section{A. 5G-PPP and NGMN Architectural Vision}

The vision for Next Generation Mobile Networks (NGMNs) is to enhance their flexible softwarization. The NGMN architecture is subdivided into three layers, as follows: i) business application, ii) infrastructure resource, and iii) its business enabling [1]. It is characterized by an End-to-End (E2E) scope encompassing the Radio Access Network (RAN) and core networks [7]. 5G Infrastructure Public-Private Partnership (5G-PPP) elaborates on roles and relationships among different parts of the $5 \mathrm{G}$ network. It generally shares the NGMN perspective that the $5 \mathrm{G}$ architecture must flexibly support softwarization for different use cases. Besides, it is worthwhile to note that the NGMN 5G-PPP architectural proposal is divided into five layers, namely service, infrastructure, business function, network function, and orchestration layers [8].

\section{B. ETSI NFV MANO Management Architecture Evolution}

NF Virtualization (NFV) enables broadening the upgraded capabilities of communication networks. These capabilities are flexible enough to instantiate the NFs where needed, e.g., in the data center or network, and provide elasticity in allocating extra resources to these NFs. However, management and orchestration functions require new algorithms that handle essential resources and control the VNFs lifecycle. To manage the VNF lifecycle and resource allocation, the ETSI has added an NFV Management and Network Orchestration (MANO) architecture. It primarily provides the management and orchestration of network services, VNF, and all resources in a data center (virtual machine resources, networking, computation, and storage) [6]. The three functional blocks of NFV MANO are i) the NFV orchestrator, ii) VNF manager, and iii) virtualized infrastructure manager [9].

\section{Slice Creation and Isolation}

Depending on use cases requirements, $5 \mathrm{G}$ heterogeneous network nature allows for designing different network slices. For example, an ultra-low-latency slice will be supported irrespectively of general network requirements if the use case requires short delays.

Slice isolation consists of creating slices and then distinguishing them by considering the use cases they will assist. Thus, it facilitates the simultaneous coexistence of multiple slices in the same network without affecting their performance. In fact, in-built security and privacy will be induced in the design by isolating the slices over the shared infrastructure [10].

\section{Standardization Activities On Network SLICING}

Standards are the guiding force behind research, development, innovation, policy establishment, and industries. It regulates the execution of productive tasks and implementation of the products while assuring quality [11]. In addition, standards ensure interoperability among research techniques or products.

There are different types of Standards Development Organizations (SDOs) working towards proposing standards within various verticals. In the context of $5 \mathrm{G}$ New Radio, it is essential to have collaborative standardization in E2E network slicing architectures [12], as it includes widespread coverage domains and application areas and is opening up new research opportunities. Therefore, SDOs and academia, together with industries, need to collaborate on standardization efforts to facilitate interoperability [11].

\section{A. Worldwide Standardization approaches on $5 G$ network slicing}

Different telecommunications standardization bodies and industries are shown in Figure 2 together with their efforts 
on network slicing a. Global System for Mobile Communications Association (GSMA) and Next Generation Mobile Network (NGMN) contribute to the investigation of high-level requirements and architecture, as well as to the creation of the concepts of E2E 5G network slicing and business initiatives [13]. Currently, the industry focuses on investigating network slicing requirements while analyzing their influence on different network layers, e.g., core network or RAN. Different SDOs have been defined as technical specifications for many domains, as follows:

- Global System for Mobile Communications Association (GSMA) is a global organization that develops a unified mobile ecosystem that supports research and innovation in the mobile communications industry sector while integrating industry solutions, including network slicing. For example, the recently published white paper on "E2E Network Slicing Architecture" [12] describes industries, operators, and vendors' requirements and the need to collaborate in standardization activities to achieve a unified solution for the NS architecture. The white paper further explains E2E NS architecture, high-level requirements, and ongoing initiatives from various SDOs.

- 3GPP, one of the main standardization bodies, encompasses several active working and study groups to support 5G network slicing. For example, the SA1 group of 3GPP focuses on use cases and requirements. The SA2 working group defines the architecture selection to support network slicing [13]. The SA3 working group addresses security while SA5 addresses slice management [14]. The NS concept was introduced in 3GPP's Release 15 [3] and has further been enhanced within Releases 16 [15] and 17 [16]. Release 16 added authentication and authorization controls, enhancements to network automation, and service-based architecture to the $5 \mathrm{G}$ slicing. Release 17 adds enhancements for phase 2 of the NS architecture and outlines support to the GSMA defined Generic Network Slice Template (GST) attributes. It also addresses simultaneous usage of network slices in 5Gassisted networks.

- The European Telecommunications Standards Institute (ETSI) activities for $5 \mathrm{G}$ network slicing address the optimization of $5 \mathrm{G}$ services, configuration, delivery, assurance in terms of deployment, enabling complete automation. It also provides a solution for computing and storage [6].

- The Internet Engineering Task Force (IETF) standardization activities address the general requirements and development of the $5 \mathrm{G}$ network slicing architecture. Besides, they consider the orchestration mechanisms and network slice management. Their latest work includes gateway function for network slicing, the applicability of abstraction, and control of traffic-engineered networks to network slicing.

- The Broadband Forum (BBF) involves the activities to term the slicing management architecture for transport networks. Moreover, the BBF standardization activities contain the sharing of broadband network infrastructure between different service providers while providing resource control support [17].

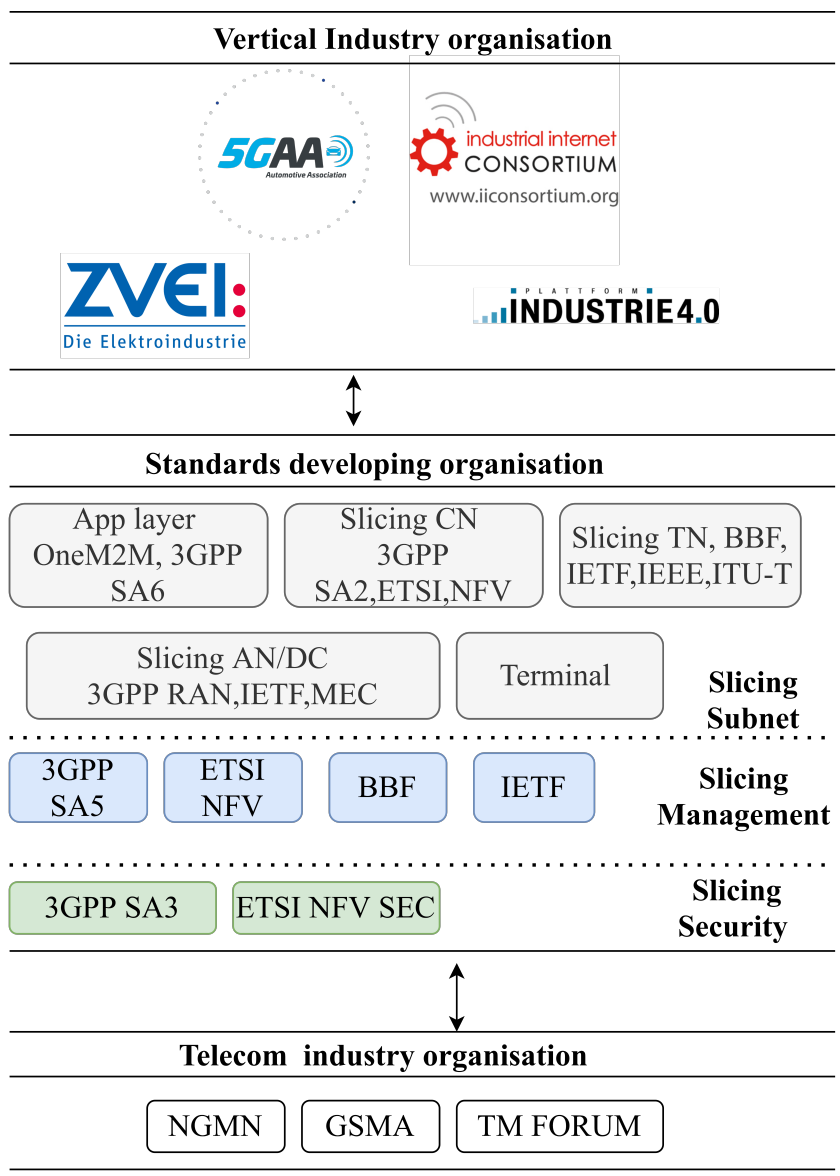

Fig. 2. Standardisation Groups and SDOs for Network Slicing [18], [19].

- The International Telecommunication Union - Telecommunication (ITU-T) encourages different functionality of E2E network slicing to provide reliability to customers. ITU-T functionality contains softwarization, network capability exposure, requirements of mobility, and diverse E2E Quality of Service (QoS) with distributed nature, the support of edge cloud, control, and user plane separations. ITU-T Study Group 13 (SG13) [20] works in orchestration, network management, and horizontal slicing standardization activities. It also addresses the data plane programmability and defines high-level network softwarization. An ITU-T SG13 Focus Group (FG) on ML for future networks, including 5G (FG-ML5G) [21], has catalogued its deliverable, which had specific requirements that include details for interfacing, network architectures, protocols, algorithms, and data formats. Deliverables related to network slicing and ML are as follows [21];

- ITU-T Y.3172 [22]: Architectural Framework for Machine Learning in Future Networks including 
IMT-2020;

- ITU-T Y.3176 [23]: ML Marketplace Integration in Future Networks including IMT-2020;

- Requirements, Architecture and Design for Machine Learning function orchestrator;

- Serving Framework for ML models in Future Networks including IMT-2020;

- Machine Learning Sandbox for Future Networks including IMT-2020: Requirements and Architecture Framework;

- Machine Learning-based End-to-End Network Slice Management and Orchestration;

- Vertical-assisted Network Slicing based on a Cognitive Framework.

- The Open Networking Foundation (ONF) studies network slicing connectivity for high bandwidth $5 \mathrm{G}$ services, by considering low latency, and secure virtual subsets of the network [24].

- The Open Network Automation Platform (ONAP) [25] is an open community by operators for next-generation network automation platform. It focuses on the unification of standards and open source activities while promoting cross-organizational collaborations. Recently, ONAP has published a series of white papers related to technical challenges associated with $5 \mathrm{G}$ slices. It emphasized on the following four design goals:

- Communication Service Template (CST) to collect SLA requirements by users;

- Service Descriptor (SD) records the user requirements collected by CST and facilitates slice creation by converting them into network requirements;

- Network Slice Template (NST) describes the deployment information of slice instances;

- Network Slice Subnet Template (NSST) deploys slice subnet instances.

\section{B. Network Slicing and $M L / A L$}

ML and AI have recently become very important in different fields of activity. It makes the system intelligent so that human intervention can be avoided. For example, Telecommunications operators use ML in analyzing the customer experience, network automation, and business process automation [19]. Additionally, ML and big data are needed to facilitate intelligent capabilities and integration for network slicing. Besides, ML and AI can be the best solution for self-optimization, self-configuration, and fault management functionalities, as well as network security and malware detection [26]. Table I presents different techniques considering ML/AI applied to the implementation of network slicing available in the literature.

\section{Opportunities For SERVICE PROVIDERS}

Mobile Network Operators (MNOs) consider network slices as an isolated independent network segment that is selfsufficient in resources and provides services at approved QoS.
TABLE I

PAPERS WITH CONCEPTS USING ML/AL FOR NETWORK SLICING.

\begin{tabular}{|l|l|}
\hline Papers & $\begin{array}{l}\text { Algorithms covered in different papers using } \\
\text { ML/AI considering Network Slicing }\end{array}$ \\
\hline$[27]$ & $\begin{array}{l}\text { Cellular and IoT networks resource management } \\
\text { techniques using ML }\end{array}$ \\
\hline$[28]$ & $\begin{array}{l}\text { Implementation of deep learning neural network, } \\
\text { considering the network availability } \\
\text { and network-load efficiency }\end{array}$ \\
\hline$[29]$ & $\begin{array}{l}\text { Network-slicing for the vehicle-to-everything service, } \\
\text { intelligent network architecture that influences the } \\
\text { recent ML techniques }\end{array}$ \\
\hline$[30]$ & $\begin{array}{l}\text { Resource mapping algorithm for 5G network slicing } \\
\text { using deep reinforcement }\end{array}$ \\
\hline$[31]$ & $\begin{array}{l}\text { A deep reinforcement learning optimization model } \\
\text { for slice configuration }\end{array}$ \\
\hline
\end{tabular}

MNOs are able to implement slicing while maintaining transparency with the end-users. NS ensures connectivity tailoredmade service delivery. MNOs maintain SLAs to administer the offered services, data rate, QoS, latency, reliability, and security. MNOs can implement either single or multiple slices in the network to offer services to varied requirements of different genres. Some of the prominent industries that can benefit from $5 \mathrm{G}$ slicing are the following ones:

- Logistics;

- Media \& Entertainment (Augmented/Virtual Reality);

- Automotive;

- Industrial Internet;

- Financial Sectors;

- Health \& Wellness;

- Smart Cities.

To meet the diverse service requirements and demands, conventional business models are evolving as well. Therefore, new use cases are being defined. The underlying market ecosystem is divided into three categories, as follows [1].

- Asset Provider: performs infrastructure leasing to the third party;

- Connectivity Provider: facilitates essential connectivity delivery at high-speed to meet QoS requirements, including latency ;

- Partner Service Provider: enables enhanced communication services to end-users \&/or to third-parties.

\section{A. Evolved Use Cases [18] [14]}

This section describes some of the evolved use cases and lists the required services from different industrial verticals.

- Massive IoT - An enormous amount of required smart services and connected devices will constitute massive IoT devices within the $5 \mathrm{G}$ evolution. These connections require high QoS and Quality of Experience (QoE). Varied applications have specific and different requirements that MNOs and CSPs can meet through network slicing. The user will pay to access network resources and functionality usage based on the SLA. 
- Automotive - Connected vehicles demand high precision with ultra-low latency requirements. Therefore, Cellular Vehicle-to-Everything (CV2X) and Vehicle-to-Everything (V2X) are considered critical use cases for 5G. Nevertheless, V2X requires communication between vehicles, emergency services, etc.; thus, these services account for high reliability. CV2X, while enabling communication among vehicles and infrastructure, creates new business opportunities, e.g., the infotainment service uses in-built sensors, cameras, and navigation modules. These services are not critical but intend to provide an enhanced driving experience. Hence, a high-speed and dedicated network is not required; instead, Mobile Broadband (MBB) connectivity will be sufficient. With network slicing, MNOs can provide ultra-low latency and high precision networks without creating a new physical dedicated network.

- Healthcare - Healthcare industries have several sub-use cases like hospital maintenance services, care-giving services, health tracking or remote monitoring. Different use cases account for different requirements. For instance, remote healthcare diagnosis requires high data rate and low latency, while hospital maintenance is non-critical.

- Industry 4.0 - Industry 4.0 defines the automation of the traditional manufacturing industries and thus, results in a large-scale connected sensors, devices, monitoring units, and intelligent productions. Different industries have different requirements, e.g., Augmented Reality (AR) can support shop-floor workers in monitoring processing flows and instruction-based tasks while in control panels with safety hazards, where human beings are involved are critical areas.

- Smart Cities - Smart cities lay down a complete infrastructure development that includes planning, deployment, management, usage, monitoring, control, and maintenance. As they aim at lifestyle enhancement and accessibility, diverse opportunities have been open in various sectors, including education, transport, basic amenities, utilities, entertainment and healthcare. Requirements vary with varying use cases. Some notable use cases include smart metering, public safety and smart street lights.

\section{Challenges And Open Research AREAS}

Network slicing is a promising paradigm for $5 \mathrm{G}$ and beyond networks, but its introduction faces various challenges [2].

In [5], NGMN has explained that sharing the resources between slice tenants is the most challenging issue for network slicing. Resource sharing can be either by static partition or by elastically dynamic sharing. One of the main open problems for resource sharing is standardizing a proper scheduling mechanism that can allocate radio resources among different slices while providing computational resource sharing and slice isolation mechanisms. Besides, network reconstruction is required in $5 \mathrm{G}$ and beyond, as the resulting ultra-dense network will comprise cooperative macro-cells and smallcells networks while addressing the slicing demands (high transmission throughput, fairness, short delay, and reliability).
Nowadays, there is no proper integration of network slicing with NFV and Cloud RAN (CRAN) to facilitate supporting point-to-point connection among radio equipment controllers and physical radio equipment. To fill this gap, network slicing requires cooperation with other $5 \mathrm{G}$ technologies, such as mobile cloud engineering, broadband transmission and NFV.

Designing new virtualization mechanisms is required to make the sharing of resources efficient and give strong support for implementing radio access network slicing. In addition, with multi-domain infrastructure, security issues turn out to be more complex. Therefore, defining security mechanism policies between several domains of the infrastructure is required.

Resource scheduling in RAN slicing is challenging due to performance isolation, diversified service requirements, and network dynamics (including user mobility and channel states).

Although different service providers and operators work on industrial solutions for network slicing and its management [32], some open management challenges include NS activation and deactivation at the service level, QoS maintenance at the network level, intra-slice resource sharing, and load balance.

NS creates separate logical networks on a shared physical infrastructure specific to use cases, realizes automation across various operational, management and business processes, and scales up the business without increasing OPEX. The commercial usage of network slicing in industries, intelligent configuration, SLA guarantees, and integration with vertical industries needs improvement. 3GPP defines the NF parameter for the slice and slice-subnet management accompanied by the related interfaces. However, it still needs further research to manage the automatic and intelligent closed-loop controls and SLA requirements. 5G network slice coordination is critical to guarantee high QoE. Use-cases and business requirements need to be considered by operators to enable new approaches that result in easy maintenance of the networks.

The open areas for future research activities include support for heterogeneous networks (HetNets) for availing intelligent services in Internet-of-Everything (IoE) scenarios, in addition to support for various kinds of realities (augmented, virtual, extended), connected autonomous systems or drone-based networks. The management and orchestration requirements vary indistinctively in drone-based networks and in conventional networks. ML and AI capabilities enable intelligent radios to support various new services while incorporating fast and efficient training, and tuning ML models. Besides, there is a need to develop novel meta-learning models for ML-enabled network slicing, an open research area.

\section{CONCLUSIONS}

In this paper, we have provided a research-based overview of the current and ongoing work on Network Slicing within different Standard Development Organizations. It includes a detailed study on application/use cases, requirements, and challenges for network slicing in the light of standardization.

In $5 \mathrm{G}$ phase- 2 and future communication generations, network slicing is expected to be one of the most influential 
technologies and provide solutions tailored to specific endusers, varying from residential to industrial or corporate. It can evolve and shift the telecommunication industry to the next level by allowing more flexible and reliable design. It is required to enhance network infrastructure and incorporates virtualization and softwarization to make the best use of services provided by network slices. It will allow operators to offer premium services to their customers. Moreover, NS will enhance the business opportunities in many sectors, which will gain attraction by increasing revenues. It is worth noting that network slicing supports the economic model and service differentiation that meets the end-user Service Level Agreements. Finally, we have identified some open issues [25] that require standardization, e.g., cross-domain inter-working, as well as SLA assurance, intelligence and automation.

\section{ACKNOWLEDGMENT}

This work was supported by FCT/MCTES through national funds and, when applicable, co-founded EU funds under the project UIDB/50008/2020, ORCIP (22141-01/SAICT/2016) and TeamUp5G. TeamUp5G has received funding from the European Union's Horizon 2020 research and innovation programme under the Marie Skłodowska-Curie ETN TeamUp5G, grant agreement No. 813391.

\section{REFERENCES}

[1] N. Alliance, "5G White Paper (Final Deliverable)," 2015.

[2] H. Zhang, N. Liu, X. Chu, K. Long, A.-H. Aghvami, and V. C. Leung, "Network slicing based 5G and future mobile networks: mobility, resource management, and challenges," IEEE communications magazine, vol. 55, no. 8, pp. 138-145, 2017.

[3] K. Flynn. (2019) A global partnership. [Online]. Available: https://www.3gpp.org/release-15

[4] "Network slicing explained," Nov 2020. [Online]. Available: https://www.nokia.com/about-us/newsroom/articles/networkslicing-explained/

[5] N. Alliance, "Description of network slicing concept," NGMN $5 G P$, vol. 1, no. 1, 2016.

[6] N. ETSI, "Network functions virtualisation (NFV); terminology for main concepts in NFV," Group Specification, Dec, 2014.

[7] X. Foukas, G. Patounas, A. Elmokashfi, and M. K. Marina, "Network Slicing in 5G: Survey and Challenges," IEEE Communications Magazine, vol. 55, no. 5, pp. 94-100, 2017.

[8] S. Redana, Ö. Bulakci, A. Zafeiropoulos, A. Gavras, A. Tzanakaki, A. Albanese, A. Kousaridas, A. Weit, B. Sayadi, B. T. Jou et al., "5G PPP architecture working group: View on 5G architecture," 2019.

[9] A. Devlic, A. Hamidian, D. Liang, M. Eriksson, A. Consoli, and J. Lundstedt, "NESMO: Network slicing management and orchestration framework," in 2017 IEEE International Conference on Communications Workshops (ICC Workshops). IEEE, 2017, pp. 1202-1208.

[10] P. Subedi, A. Alsadoon, P. Prasad, S. Rehman, N. Giweli, M. Imran, and S. Arif, "Network slicing: a next generation 5G perspective," EURASIP Journal on Wireless Communications and Networking, vol. 2021, no. 1, pp. 1-26, 2021.

[11] N. Abdelkafi, R. Bolla, C. J. Lanting, A. Rodriguez-Ascaso, M. Thuns, and M. Wetterwald, "Understanding ICT standardization: Principles and practice," 2019.

[12] GSMA. (2021) E2E Network Slicing Architecture Version 1.0. [Online]. Available: https://www.gsma.com/newsroom/wpcontent/uploads//NG.127-v1.0-2.pdf

[13] P. Rost, C. Mannweiler, D. S. Michalopoulos, C. Sartori, V. Sciancalepore, N. Sastry, O. Holland, S. Tayade, B. Han, D. Bega et al., "Network slicing to enable scalability and flexibility in 5G mobile networks," IEEE Communications Magazine, vol. 55, no. 5, pp. 72-79, 2017.
[14] X. De Foy and A. Rahman, "Network slicing-3GPP use case," Working Draft, IETF Secretariat, Internet-Draft draft-defoy-netslices-3gppnetwork-slicing-02, 2017.

[15] K. Flynn, "A global partnership," Mar 2020. [Online]. Available: https://www.3gpp.org/release-16

[16] _ "A global partnership," Dec 2020. [Online]. Available: https://www.3gpp.org/release-17

[17] C.-Y. Chang and N. Nikaein, "Closing in on 5G control apps: enabling multiservice programmability in a disaggregated radio access network," IEEE vehicular technology magazine, vol. 13, no. 4, pp. 80-93, 2018.

[18] GSMA. (2018) Use Case Requirements. [Online]. Available: https://www.gsma.com/futurenetworks/resources/network-slicinguse-cases-requirements-2/.

[19] S. Wijethilaka and M. Liyanage, "Survey on network slicing for Internet of Things realization in 5G networks," IEEE Communications Surveys \& Tutorials, vol. 23, no. 2, pp. 957-994, 2021.

[20] ITU-T. (2019) Progress of 5G studies in ITU-T: overview of SG13 standardization activities . [Online]. Available: https://www.itu.int/en/ITUT/Workshops-and-Seminars/20180604/ Documents/Session1.pdf

[21] "Focus Group on Machine Learning for Future Networks including 5G." [Online]. Available: https://www.itu.int/en/ITU$\mathrm{T} /$ focusgroups $/ \mathrm{ml} 15 \mathrm{~g} / \mathrm{Pages} / \mathrm{default}$.aspx

[22] ITU-T, "Architectural framework for machine learning in future networks including imt-2020," Jun 2019. [Online]. Available: https://www.itu.int/rec/T-REC-Y.3172/en

[23] - "Machine learning marketplace integration in future networks including imt-2020," Oct 2020. [Online]. Available: https://www.itu.int/rec/T-REC-Y.3176-202009-P

[24] ONF. (2019) Transport API (TAPI) 2.0 Overview Version 0.0 August. [Online]. Available: https://opennetworking.org/wpcontent/uploads/2017/08/TAPI-2-WP_DRAFT.pdf

[25] L. Deng, H. Deng, and A. Mayer. (2021) Harmonizing Open Source and Standards: A Case for 5G Slicing. [Online]. Available: https://www.onap.org/wp-content/uploads/sites/20/2020/03/ ONAP_HarmonizingOpenSourceStandards_031520.pdf

[26] L. Xiao, X. Wan, X. Lu, Y. Zhang, and D. Wu, "IoT security techniques based on machine learning: How do IoT devices use AI to enhance security?" IEEE Signal Processing Magazine, vol. 35, no. 5, pp. 41-49, 2018.

[27] F. Hussain, S. A. Hassan, R. Hussain, and E. Hossain, "Machine learning for resource management in cellular and IoT networks: Potentials, current solutions, and open challenges," IEEE Communications Surveys \& Tutorials, vol. 22, no. 2, pp. 1251-1275, 2020.

[28] A. Thantharate, R. Paropkari, V. Walunj, and C. Beard, "DeepSlice: A deep learning approach towards an efficient and reliable network slicing in 5G networks," in 2019 IEEE 10th Annual Ubiquitous Computing, Electronics \& Mobile Communication Conference (UEMCON). IEEE, 2019, pp. 0762-0767.

[29] J. Mei, X. Wang, and K. Zheng, "Intelligent network slicing for V2X services toward 5G," IEEE Network, vol. 33, no. 6, pp. 196-204, 2019.

[30] L. Zhao and L. Li, "Reinforcement learning for resource mapping in 5G network slicing," in 2020 5th International Conference on Computer and Communication Systems (ICCCS). IEEE, 2020, pp. 869-873.

[31] S. De Bast, R. Torrea-Duran, A. Chiumento, and H. Pollin, Sofie and Gacanin, "Deep reinforcement learning for dynamic network slicing in IEEE 802.11 networks," in IEEE INFOCOM 2019-IEEE Conference on Computer Communications Workshops (INFOCOM WKSHPS). IEEE, 2019, pp. 264-269.

[32] M. Jiang, M. Condoluci, and T. Mahmoodi, "Network slicing management \& prioritization in $5 \mathrm{G}$ mobile systems," in European Wireless 2016; 22th European Wireless Conference. VDE, 2016, pp. 1-6. 